\title{
Detection of a Novel, and Likely Ancestral, Tn916-Like Element from a Human Saliva Metagenomic Library
}

\author{
Liam J. Reynolds ${ }^{1,2}$, Muna F. Anjum ${ }^{2}$ and Adam P. Roberts ${ }^{3, *(D)}$ \\ 1 UCD School of Biomolecular and Biomedical Science, University College Dublin, 4 Dublin, Ireland; \\ liam.reynolds@ucd.ie \\ 2 Department of Bacteriology, Animal and Plant Health Agency, Addlestone KT15 3NB, UK; \\ muna.anjum@apha.gov.uk \\ 3 Department of Tropical Disease Biology, Liverpool School of Tropical Medicine, Pembroke Place, \\ Liverpool L3 5QA, UK \\ * Correspondence: Adam.Roberts@lstmed.ac.uk
}

Received: 12 April 2020; Accepted: 12 May 2020; Published: 14 May 2020

\begin{abstract}
Tn916 is a conjugative transposon (CTn) and the first reported and most well characterised of the Tn916/Tn1545 family of CTns. Tn916-like elements have a characteristic modular structure and different members of this family have been identified based on similarities and variations in these modules. In addition to carrying genes encoding proteins required for their conjugation, Tn916-like elements also carry accessory, antimicrobial resistance genes; most commonly the tetracycline resistance gene, tet(M). Our study aimed to identify and characterise tetracycline resistance genes from the human saliva metagenome using a functional metagenomic approach. We identified a tetracycline-resistant clone, TT31, the sequencing of which revealed it to encode both tet $(\mathrm{M})$ and tet $(\mathrm{L})$. Comparison of the TT31 sequence with the accessory, regulation, and recombination modules of other Tn916-like elements indicated that a partial Tn916-like element encoding a truncated orf9 was cloned in TT31. Analysis indicated that a previous insertion within the truncated orf9 created the full length orf9 found in most Tn916-like transposons; demonstrating that orf9 is, in fact, the result of a gene fusion event. Thus, we hypothesise that the Tn916-like element cloned in TT31 likely represents an ancestral $\operatorname{Tn} 916$.
\end{abstract}

Keywords: saliva metagenome; Tn916; antibiotic resistance; orf9; tetracycline resistance; integrative conjugative element

\section{Introduction}

Tn916 is the archetype of the Tn916/Tn1545 family of broad host range conjugative transposons or integrative and conjugative elements (CTns and ICEs respectively), members of which have been described in over 35 genera of bacteria [1-3]. CTns, or ICEs, are mobile genetic elements that can mediate their own transfer from one cell to another via direct cell to cell contact and a type IV secretion system dependent conjugation process. They carry all the necessary genes that encode for the proteins required for their excision from (and integration into) the host genome, their conjugal transfer to a recipient cell and, as recently proven, their independent replication as an excised circular molecule [4,5]. Tn916-like elements have modular structures; they contain characteristic conjugation, regulation modules and usually a tyrosine integrase comprising a recombination module plus more variable accessory genes that almost always contain antimicrobial resistance genes; primarily tet $(\mathrm{M})$. The gene tet $(\mathrm{M})$ encodes a ribosomal protection protein conferring resistance to tetracyclines although genes conferring resistance to antibiotics other than tetracycline such as 
macrolides (erm(B); accession no. AM410044), biocides ( $q r g ;$ accession no. HQ663849) and heavy metals (merA; accession no. EU239355) have also been identified on Tn916-like elements [1,3,6,7]. Although the genes present in these modules can differ between different members of this family their modular structure can still be used as a basis to identify them $[3,8]$.

The array of accessory genes identified on Tn916-like elements is evidence of the continuing gene acquisition events that play an important role in the evolution of this CTn family. For example, erm(B) is associated with IS1216 insertion sequences (ISs) on Tn1116 [9]. In fact, IS1216 transposases have shaped the structure of other Tn916-like elements including Tn6087 and Tn6079 by introducing qrg and $\operatorname{erm}(\mathrm{T})$ antimicrobial resistance genes respectively onto a Tn916 backbone [1,10]. Exchanges between Tn916-like elements and introns, plasmids and other transposons have also been described [11-13]. For example; both Tn5397 and Tn6000 carry a group II intron inserted into orf14 and so this insertion may be a point of divergence between these two CTn's and the Tn916 progenitor which does not contain a group II intron $[14,15]$. Genetic reorganization events have not been limited to just the acquisition of accessory genes. Genes involved in the regulation of conjugation vary between Tn916-like elements. For example, CTn1 from Clostridioides difficile and Tn5386 do not encode orf9, orf10, orf5 and orf6. This further demonstrates that gene acquisition and deletion play a role in the Tn916-like element evolution. It also suggests that different Tn916-like elements employ different regulatory pathways despite the central role as a transcriptional repressor being proposed for orf9 [16,17].

Evolution of other CTn families via gene acquisition, deletion and recombination have also been reported. Wozniak et al. demonstrated that there is a minimum gene structure required for SXT/R391-type conjugal transfer and that acquisition of genes within five "hotspots" of this core structure shaped the evolution and diversity of this CTn family [18]. Similarly, CTn's of the ICESt1/ICESt3 family have a defined core structure and a variable region where gene acquisition has been described. ICESt1 and ICESt3 differ in that the former encodes a bacteriophage resistance system in its variable region and the latter encodes putative methyltransferase genes [19,20].

Multiple studies have reported the identification of antibiotic-resistant bacteria and resistance genes from the human oral cavity. Indeed, the association of antibiotic resistance genes with Tn916-like elements from oral bacteria, in particular Streptococcus spp., has been described [21,22]. As estimates indicate that only two-thirds of the bacteria from this environment have been cultured we created and functionally screened, a human saliva metagenomic library to identify antibiotic resistance genes and associated mobile genetic elements [23]. We have previously reported on the novel efflux genes tet $\mathrm{AB}(60)$ encoding resistance to tetracycline and tigecycline in Escherichia coli and multiple genes encoding resistance to biocides from this metagenomic library [24,25].

In this manuscript, we report on a tetracycline-resistant clone identified in this metagenomic library designated TT31. It contains both tet(M) and tet(L), encoding a tetracycline efflux gene. Both genes are part of a novel Tn916-like element. Analysis of the clone indicates that the DNA likely originated from a host that shares sequence homology to Gemella sp. that also harbors what we propose to be an ancestral Tn916-like element.

\section{Materials and Methods}

\subsection{Bacteria Strains and Culture Conditions}

E. coli EPI300 clones were cultured in Luria-Bertani broth (LB; Sigma-Aldrich ${ }^{\circledR}$, Gillingham, UK) and LB agar (Life Technologies ${ }^{\mathrm{TM}}$, Renfrew, UK) at $37^{\circ} \mathrm{C}$ with shaking at $200 \mathrm{rpm}$ for liquid cultures. Antibiotic selection was achieved by supplementing media with chloramphenicol $(12.5 \mu \mathrm{g} / \mathrm{mL}$; Sigma-Aldrich $\left.{ }^{\circledR}\right)$ and tetracycline $\left(5 \mu \mathrm{g} / \mathrm{mL}\right.$; Sigma-Aldrich $\left.{ }^{\circledR}\right)$.

\subsection{Creation of a Functional Metagenomic Library}

Metagenomic DNA was extracted from the pooled saliva of 11 individuals (in $1.5 \mathrm{~mL}$ aliquots) who had not received antibiotics within the previous 3 months. The UCL Research Ethics Committee 
granted ethical approval to collect human saliva from volunteers (Project ID Number 5017/001) and all participants gave written consent before participating. The human saliva metagenomic library was created using the pCC1BAC vector and the tetracycline sensitive (minimum inhibitory concentration of $2 \mu \mathrm{g} / \mathrm{mL}$ ) strain TransforMax EPI300 Electrocompetent E. coli as previously described [24,25].

\subsection{Screening of the Functional Metagenomic Library}

From the human saliva functional metagenomic library, 27,000 clones were screened for tetracycline resistance by culturing the clones on LA supplemented with chloramphenicol $(12.5 \mu \mathrm{g} / \mathrm{mL}) \mathrm{and}$

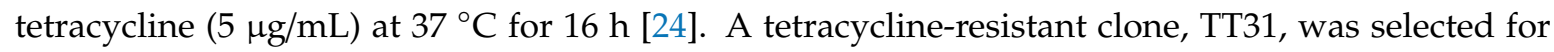
further characterisation.

\subsection{Plasmid Extraction}

In $20 \mathrm{~mL}$ tubes, $1 \mathrm{~mL}$ of a $16 \mathrm{~h}$ culture of TT31 in LB supplemented with chloramphenicol $(12.5 \mu \mathrm{g} / \mathrm{mL})$ and tetracycline $(5 \mu \mathrm{g} / \mathrm{mL})$ was subcultured into $9 \mathrm{~mL}$ fresh LB supplemented with chloramphenicol $(12.5 \mu \mathrm{g} / \mathrm{mL})$, tetracycline $(5 \mu \mathrm{g} / \mathrm{mL})$ and $10,000 X$ CopyControl $^{\mathrm{TM}}$ Induction solution (10 $\mu \mathrm{L}$ in $10 \mathrm{~mL}$ ). This subculture was then incubated horizontally (by taping the tube, on its side, to the base of the incubator) for $4 \mathrm{~h}$ at $37^{\circ} \mathrm{C}$ with shaking at $200 \mathrm{rpm}$. The pCC1BAC vector was extracted from this TT31 clone subculture using the QIAprep Spin Miniprep Kit (QIAGEN, Manchester, UK) according to the manufacturer's guidelines.

\subsection{Sequencing and Analysis}

The pCC1BAC based plasmid extracted from TT31 was sequenced using primer extension Sanger sequencing by Beckman Coulter Genomics Inc using the primers detailed in Table S1. Assembly of the TT31 contig was conducted using SeqMan Pro (Lasergene software, DNASTAR, Madison, WI, USA). The TT31 sequence was submitted to GenBank (accession: MF344584) and is included in Figure S1. The sequences were analysed using the tools from the National Centre for Biotechnology Information (NCBI). Additional sequence data of interest were obtained from the NCBI database and alignments were made using the Clustal $\Omega$ software at http://www.ebi.ac.uk/Tools/msa/clustalo/.

\section{Results}

\subsection{Sequence Analysis and Gene Annotation of TT31 Insert}

Sequencing of the pCC1BAC::vector insert extracted from TT31 revealed it to be 14,171 bp in size. BLASTN analysis of the sequence showed that from $1 \mathrm{bp}$ to $7226 \mathrm{bp}$ (the left side of Figure 1a) had 98\% nucleotide identity to Streptococcus pyogenes strain emm65 (99\% cover; accession: CP035433). The left side of TT31 encoded nine ORFS including an excisionase (xisTn), a tyrosine integrase (intTn), tet(M) as well as a variant of orf9, orf5, orf8, orf7 and orf10 all of which are found on Tn916-like elements. An ORF exhibiting $99 \%$ nucleotide identity to tet(L) from several Enterococcus and Streptococcus species was also identified (Figure 1a,b).

TT31, from $7214 \mathrm{bp}$ to $14,171 \mathrm{bp}$, (the right side, Figure $1 \mathrm{a}$ ), had $95 \%$ nucleotide identity to Gemella haemolysans NCTC 10459 (accession:LR134484.1). This region of TT31 encoded yheH and yheI (multidrug ABC transporter subunits), a NAD-dependent deacetylase and a SecA translocase; all of which have homology to proteins from G. haemolysans NCTC 10459 (>98\%), (Figure 1a).

As HindIII had been used to digest the oral metagenomic DNA for library construction we ascertained the position of any internal HindIII site to rule out the possibility of concatemer formation during cloning. No HindIII site was present at the boundary of the left side (exhibiting homology to S. pyogenes strain emm65) and the right side (exhibiting homology to G. haemolysans NCTC 10459) of TT31, indicating that the insert was not a concatemer and that it wasn't a result of our cloning protocol. 
(a) Left

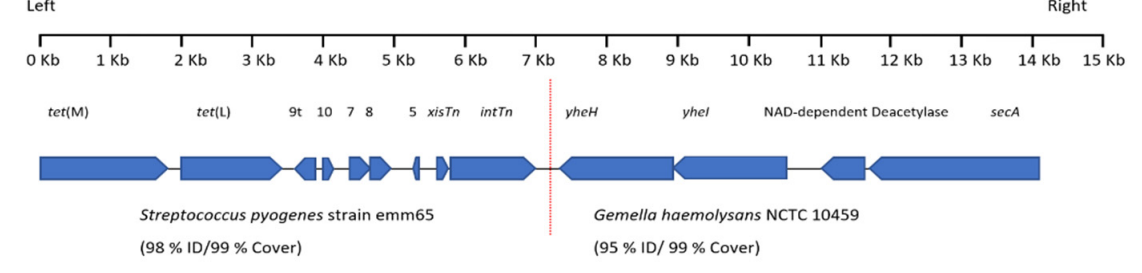

(b)

$0 \mathrm{~Kb} \quad 1 \mathrm{~Kb} \quad 2 \mathrm{~Kb} \quad 3 \mathrm{~Kb} \quad 4 \mathrm{~Kb} \quad 5 \mathrm{~Kb} \quad 6 \mathrm{~Kb} \quad 7 \mathrm{~kb}$
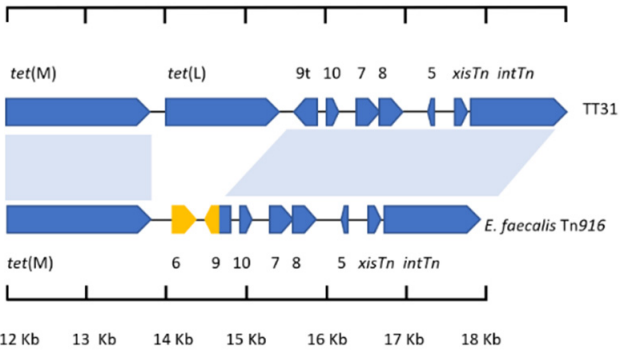

(c)

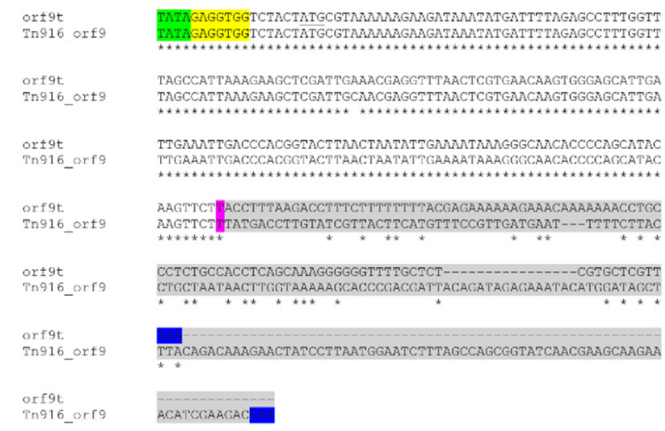

(d)

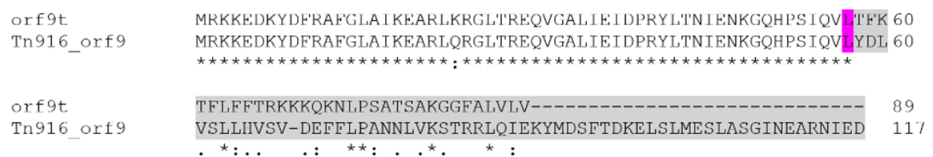

Figure 1. Alignment of TT31 and Tn916. (a) A schematic of the ORFs present on the TT31 Clone. The vertical dashed red line highlights the border of the sequences with nucleotide homology to Streptococcus pyogenes strain emm65 on the left and on the right Gemella haemolysans NCTC 10459. orf9t, orf10, orf7, orf8, and orf5 are shown as 9t, 10, 7, 8 and 5 respectively. (b) TT31 and Tn916 with aligned regions indicated by blue shaded areas. Regions of Tn916 that are not present in TT31 are highlighted in yellow. tet $(\mathrm{M})$ is found in TT31 and Tn916 as are the Tn916 regulation and recombination modules. tet $(\mathrm{L})$, however, is not present on Tn916 and orf6 is not encoded by TT31. Furthermore, orf9 is truncated in TT31 (designated orf9t) compared to Tn916 orf9. (c) A Clustal $\Omega$ alignment between orf9t and Tn916 orf9. The start codons are underlined and stop codons are highlighted blue. The predicted 'TATA box' and Shine-Dalgarno sequences are highlighted green and yellow respectively for both. The purple highlighted nucleotides identify the point of divergence between orf 9 and orf $9 t$. The grey shaded area represents the 99 nucleotides and 183 nucleotides of the $3^{\prime}$-end of orf9t and orf9 respectively that do not share homology. The stop codon for both are highlighted blue. (d) A Clustal $\Omega$ alignment between the amino acid sequences of Orf9t and Tn916 Orf9. The purple highlighted amino acids represent the point of divergence of homology between the two proteins and represents the same point of divergence observed for the nucleotide alignment. The grey shaded amino acids are those that share no homology at the N-terminal end of both proteins. 


\subsection{Nucleotide Sequence Homology of the TT31 Insert with Tn916}

BLASTN analysis revealed Tn916 from Enterococcus faecalis (accession: U09422) to have 74\% coverage and $97 \%$ nucleotide identity to the left 7226 bp of TT31 (Figure 1b) [8]. An alignment between $1 \mathrm{bp}$ and $7226 \mathrm{bp}$ of TT31 and the $5844 \mathrm{bp}$ regulatory, recombination and accessory [tet(M)] modules of E. faecalis Tn916 showed that the first $1874 \mathrm{bp}$ of TT31 aligned with the E. faecalis $\operatorname{Tn} 916$ tet(M) (12,185 bp-14,058 bp). Between 3759 bp to $7216 \mathrm{bp}$, TT31 had $97 \%$ nucleotide identity to E. faecalis Tn916 (14,571 bp-18,032 bp) (Figure 1b). This region of TT31 encoded orf 10, orf7, orf 8, orf5, the xisTn and intTn genes as well as the $5^{\prime}$-part of orf9, that are characteristic of Tn916 [22,26]. Within TT31, no alignment was observed between $1875 \mathrm{bp}-3759 \mathrm{bp}$ and Tn916, this region contained tet $(\mathrm{L})$ which is not encoded by Tn916; this explains the $74 \%$ coverage observed for this alignment.

\subsection{TT31 Encodes a Truncated orf9}

Interestingly, TT31 orf9, which we have designated orf9t, was truncated compared to that found on Tn916. The Tn916 orf9 is an open reading frame of 354 nucleotides whereas orf9t (270 nucleotides) was 84 nucleotides shorter at the $3^{\prime}$ - end. The first 171 nucleotides of the Tn916 orf9 showed $>99 \%$ nucleotide identity with TT31 orf9t; the nucleotides following this point shared no homology (Figure 1c). The lack of homology between the $3^{\prime}$-ends of orf9t and Tn916 orf9 was further demonstrated by aligning their putative amino acid sequences. Orf9t and Tn916 Orf9 showed no amino acid homology after amino acid 57, which agreed with the nucleotide alignment (Figure 1d). Additionally, 14,058 bp-14,570 bp of Tn916, which encoded orf6, did not align with TT31 (Figure 1b). BLAST analysis of the orf9t sequence revealed it to be entirely present in the regulatory region of Tn916-like elements in S. pyogenes strain emm65, S. pyogenes NCTC 13737 (LS483425.1) and Streptococcus agalactiae strain 874,391 (CP022537.1).

\subsection{A Plasmid Insertion Occurred within orf9t in Lactobacillus Johnsonii}

Analysis of the first 7226 bp of TT31 revealed it to have $60 \%$ coverage and $97 \%$ nucleotide identity to a 12,313 bp section of the Lactobacillus johnsonii strain BS15 genome that encodes tet(L) (accession: CP016400). An alignment of this 7226 bp region of TT31 with the $12,313 \mathrm{bp}$ region of the L. johnsonii strain BS15 genome showed that there is an insertion in the L. johnsonii sequence relative to TT31 which explains why there is only $60 \%$ coverage between the two sequences. This insertion is in orf $9 t$, between nucleotides $3758 \mathrm{bp}$ and $3759 \mathrm{bp}$ as these nucleotides aligned to positions 41,612 bp and 46,612 bp respectively on the L. johnsonii genome (Figure 2). Four ORFs were found to be encoded in this intervening $4999 \mathrm{bp}$ section of DNA. These ORFs encoded pre/mob and rep genes, an IS110 family transposase and an orf 6 homolog. The $183 \mathrm{bp}$ of the $3^{\prime}$ - end of orf9 that is present on Tn916 but not in TT31 was also contained in this insert (Figure 2). This $3^{\prime}$ - end of orf9 was in frame with the start codon containing $5^{\prime}$ - end of orf9t such that L. johnsonii strain BS15, like Tn916, encoded the full length fusion orf9 (Figure 2). The 99 bp $3^{\prime}$ - end of orf9t was retained by the L. johnsonii genome, outside of the insertion (Figure 2).

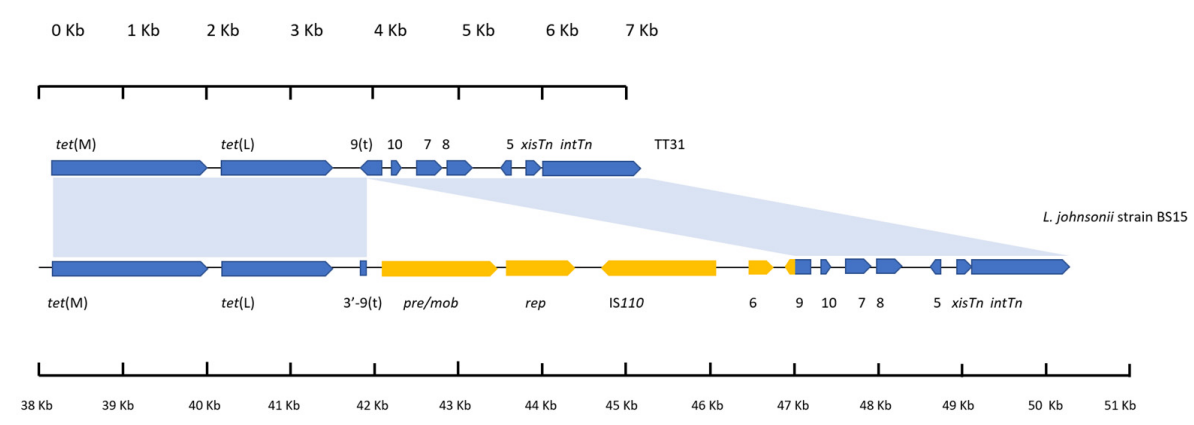

Figure 2. An alignment of the left $7226 \mathrm{bp}$ of TT31 and a 12,313 bp region of the L. johnsonii strain 
BS15 genome. Blue shaded areas represent areas of alignment between the two sequences. Individual scales accompany each gene schematic. tet $(\mathrm{M})$ and tet $(\mathrm{L})$ are encoded by both as are orf10, orf7, orf8, orf5, xisTn and intTn. orf9t is encoded by TT31. An insertion is present in the orf9t of L. johnsonii strain BS15 relative to TT31. pre/mob, rep, IS110 transposase and orf6 genes are present on this insert; highlighted in yellow. Also present on this insert is the $183 \mathrm{bp} 3^{\prime}$ - end of the full length orf9 (highlighted in yellow) in frame with the start codon of the $5^{\prime}$ end of orf 9 that is found in all the sequences (highlighted in blue); thus the full length orf9 is encoded by L. johnsonii strain BS15. The L. johnsonii genome still maintains the $3^{\prime}$ - end of orf9t (highlighted blue).

\section{5. tet $(L)$ and the 3'-orf9t Sequence Are Present in the TT31 Tn916-Like Element and Tn6079}

Tn6079 (accession: GU951538) is a CTn encoded by Streptococcus gallolyticus that was first identified from a functional metagenomic screen of the infant gut [10]. As Tn6079 encoded tet $(\mathrm{L})$ and had a similar genetic organisation to TT31, the left $7226 \mathrm{bp}$ of TT31 was aligned with the 11,701 bp accessory, regulation and recombination modules of Tn6079. This revealed a $99 \%$ nucleotide identity ( $82 \%$ cover) between the two sequences (Figure 3). Alignment of TT31 (between $1 \mathrm{bp}$ and $3758 \mathrm{bp}$ ) and Tn6079 (between 17,176 bp and 20,917 bp) represents homology between the tet $(\mathrm{M})$ and tet $(\mathrm{L})$ encoding sections of the sequences. It also confirms that the $99 \mathrm{bp} 3^{\prime}$-end of orf9t is present on Tn6079. The orf5, xisTn and intTn encoding regions of TT31 (between $4993 \mathrm{bp}$ and $7226 \mathrm{bp}$ ) and Tn6079 (between 26,639 bp and $28,876 \mathrm{bp}$ ) also aligned (97\% nucleotide identity) (Figure 3). The orf8, orf7, orf10 and the $5^{\prime}$-end of orf9t encoding region of TT31 (3759 bp-4993 bp) did not align with Tn6079. Additionally, the accessory Tn6079 genes encoding a plasmid recombination protein ( $\mathrm{pre} / \mathrm{mob}$ ), a plasmid replication protein (rep) and $\operatorname{erm}(\mathrm{T})$ that is flanked by IS1216 sequences (20,918 bp-26,639 bp) did not align with TT31, (Figure 3). The pre/mob and rep genes of Tn 6079 had $>99 \%$ nucleotide identity to those identified in the L. johnsonii strain BS15 genome insertion.

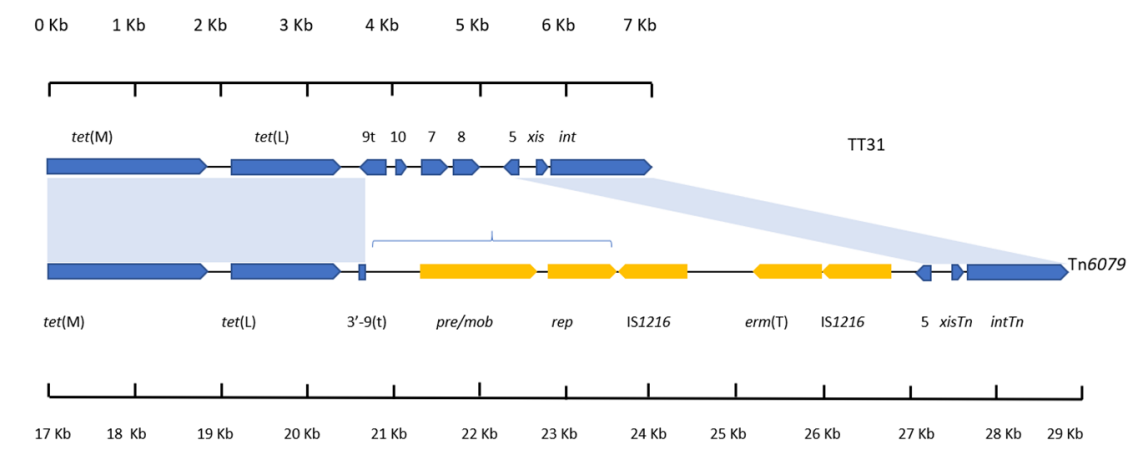

Figure 3. An alignment between the left $7226 \mathrm{bp}$ of TT31 and the right 11,701 bp of Tn6079. Regions of Tn6079 and TT31 that aligned are indicated by the blue shaded areas. Individual scales accompany each gene schematic. tet(M) and tet(L) are present in both as are orf5, xisTn and intTn. orf9, orf10, orf7 and orf8 are not present on Tn6079. The pre/mob, rep and erm $(\mathrm{T})$ genes (the latter of which is flanked by IS1216 sequences) that are encoded by Tn6079 are not present on TT31 and are highlighted in yellow. The region of Tn6079 highlighted by blue brackets is present in the L. johnsonii BS15 genome also.

\section{Discussion}

\subsection{A Partial Tn916-Like Element Was Cloned in TT31}

Analysis of the TT31 insert showed the left 7226 bp had homology to a Tn916-like element from $S$. pyogenes strain emm65 and the right side of the TT31 insert had homology to G. haemolysans NCTC 10459. The lack of HindIII restriction site between the left and right sides of the cloned insert indicated that it was not a concatemer. Therefore, the cloned insert contained a partial Tn916-like element from a host sharing homology with a Gemella sp. Tetracycline resistance mediated by tet $(\mathrm{M})$ has been described in Gemella spp. before; evidence for its association with the Tn916 integrase (intTn) in this genus was 
reported when Zolezzi et al. [27] used PCR to identify these markers in nasopharyngeal Gemella spp. isolates, however, beyond this study little work has been conducted regarding Tn916-like elements in this genus [27-29]. To the best our knowledge tet(L) encoding Gemella spp. have not been described in the literature. However, it should be noted that sequence analysis of the small section of DNA cloned in TT31 is not sufficient to identify the exact genera from which the cloned DNA originated and so it is possible the DNA originated from a non-Gemella sp.

\subsection{The Impact of tet(L) Expression on a Tn916-Like Element}

Transcription of tet $(\mathrm{M})$ and surrounding genes are proposed to play an important role in the regulation of Tn916 excision and integration. In the absence of tetracycline, tet $(\mathrm{M})$ transcription is hypothesised to be prevented by stem-loop structures in the upstream, orf12 RNA that halts the progress of the RNA polymerase through tet(M) and beyond [30]. In the presence of subinhibitory concentrations of tetracycline most ribosomes are inhibited and so charged tRNA molecules accumulate within the cell. Under these conditions, some ribosomes are thought to be protected by basal levels of Tet(M). These protected ribosomes can rapidly transcribe the orf12 transcript as charged tRNA molecules are more readily available and this prevents stem-loop structures forming, resulting in increased tet $(\mathrm{M})$ transcription as the RNA polymerase is no longer halted. Increased transcription through tet(M) is proposed to produce antisense orf9 RNA. Orf9 has putative DNA binding domains and is believed to repress transcription of orf7; an up-regulator of xisTn and intTn expression. As such the production of antisense orf9 RNA de-represses orf7 resulting in excision of the Tn916 element from the host genome, circularisation of the excised element and increased transcription, across the ligated ends of the element, of the conjugation related genes on the other end of the CTn [31,32].

In the context of the TT31 Tn916-like element, transcription through tet(M) as described above would also result in increased transcription of tet $(\mathrm{L})$ which is immediately downstream of it. Tet(L) is an antiporter of the major facilitator superfamily that pumps tetracycline out of the cell using the proton motive force generated by the concomitant uptake of a proton [33,34]. Thus, increased expression of Tet(L) could act to reduce tetracycline levels in the cell below the threshold concentration required to initiate excision of the CTn as described above. Tet(L) could, therefore, act as a second gauge that controls the excision of the CTn under unfavorable conditions, such as low $\mathrm{pH}$, that oral bacteria frequently encounter [35]. Such a scenario would offer some explanation as to why tet(L) has been identified downstream of tet(M) in only a few Tn916-like elements as the excision of such elements could be more tightly regulated and therefore, they may have disseminated less. The loss of tet $(\mathrm{L})$ could have loosened this control allowing CTn's expressing only tet(M) to excise and disseminate more readily.

The role of antisense orf9 production and its role in regulating the excision of tet(M) and tet(L) encoding Tn916-like elements requires further study. To date Tn6079 is the only tet(M) and tet $(\mathrm{L})$ encoding Tn916 element that has been described in detail. Tn6079 does not encode orf9, however, and so regulation of its excision is by some alternative pathway [10].

The maintenance of tet(L) in addition to tet(M) by some Tn916-like elements may be a result of tet(L) expression offering some additional benefit to the host cell. Indeed, Tet(L) has been shown to accept $\mathrm{Na}^{+}$and $\mathrm{K}^{+}$as substrates and may act as an important regulator of these ions under conditions of stress [36]. For example, B. subtilis strains in which tet $(\mathrm{L})$ has been deleted are more sensitive to high $\mathrm{pH}$ than their wild type tet(L) expressing counterparts [37].

\subsection{The TT31 Insert Contained a Partial 'Ancestral' Tn916-Like Element}

The results of our analysis indicated that TT31 contained a partial and likely "ancestral" Tn916 element and that a series of hypothetical events of acquisition and subsequent loss of genes by this 'ancestral' transposon culminated in the formation of Tn916. In this evolutionary scenario, the Tn916-like element encoded by the L. johnsonii strain BS15 genome is representative of an 'intermediate' between Tn916 and the TT31 Tn916-like element. The series of events may have taken place as 
follows. A pre/mob, rep, transposase and orf6 encoding plasmid may have inserted into orf9t of the TT31 Tn916-like element. This plasmid also contained the $3^{\prime}-183 \mathrm{bp}$ of the full length orf9 gene, from Tn916, and its insertion into the TT31 Tn916-like element resulted in the creation of the Tn916-like element identified in L. johnsonnii strain BS15. Staphylococcus aureus strain ADB2006 (MH8232.16) carries a pathogenicity island that encodes sequences with $75 \%$ and $85 \%$ nucleotide identity to Tn916 orf6 and the $3^{\prime}-183 \mathrm{bp}$ of full length orf9, respectively. The orf6 and the $3^{\prime}-183 \mathrm{bp}$ of the full length orf9 may have been acquired by the pre/mob, rep, transposase encoding plasmid prior to insertion in the TT31 Tn916-like element (Figure 4). The existence of these sequences outside of a Tn916-like element supports our hypothesis that the full length Tn916 orf9 is the result of a gene fusion that resulted from an insertion event into orf9t.

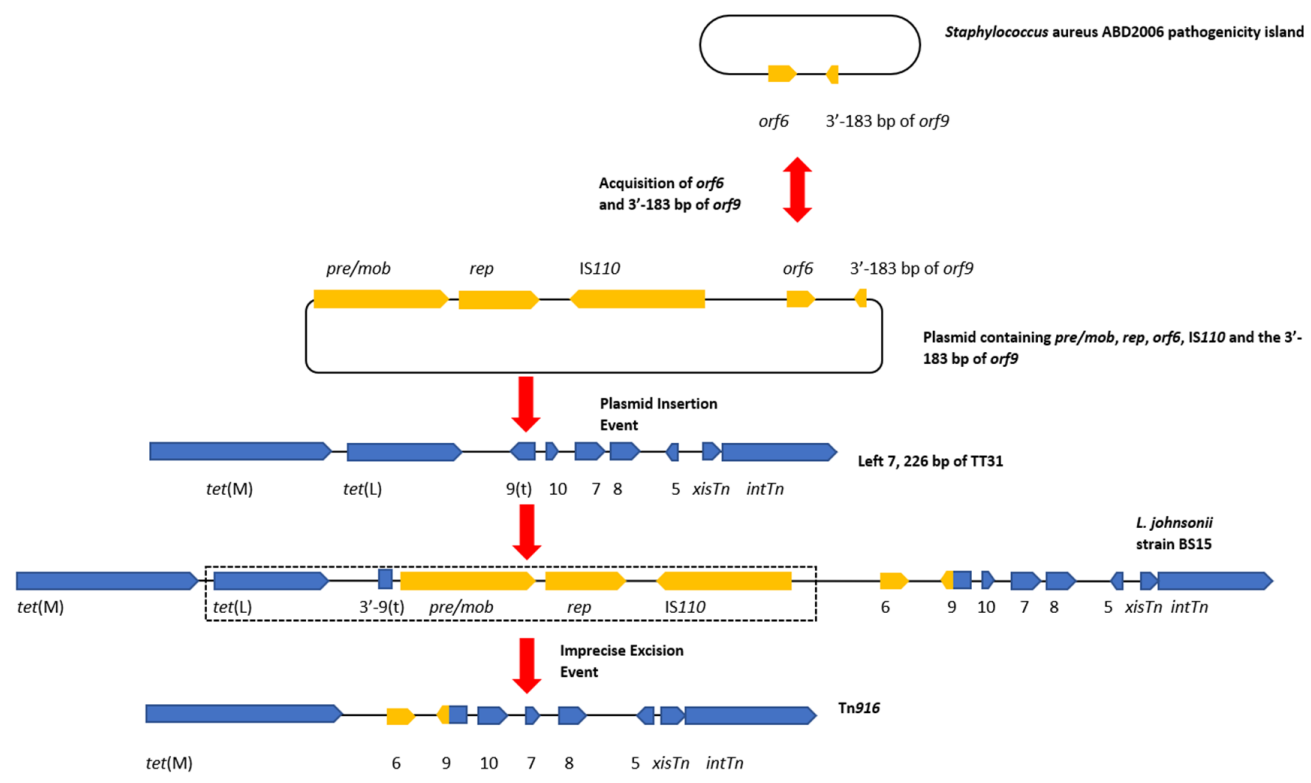

Figure 4. A diagram of the hypothetical events that led to the creation of Tn916. This hypothetical event assumes that the left 7226 bp of TT31 are a part of a larger Tn916-like element. Gene diagrams are not to scale. A plasmid containing pre/mob and rep genes and an IS110 transposase obtains orf6 and the 3' - end of orf9 (yellow) from the Staphylococcus aureus ABD2006 pathogenicity island. A double-headed arrow between the Staphylococcus aureus ABD2006 pathogenicity island and the plasmid indicates that orf6 and the $3^{\prime}-183$ bp of orf9 may have transferred in either direction. Both scenarios demonstrate the mobility of this region of DNA. Insertion of this plasmid then occurs within orf9t of the TT31 Tn916-like element which creates the L. johnsonii strain BS15 encoded 'intermediate' Tn916-like element. This plasmid insertion disrupts the orfgt reading frame resulting in the creation of the larger fusion orf 9 (yellow and blue) although the 3'-end of orf9t is still present in L. johnsonii strain BS15. An imprecise excision event then occurs which results in the loss of the pre/mob, rep, IS110 transposase and tet(L) genes as well as the $3^{\prime}$ - end of orf9t (indicated by a dotted box), creating Tn916.

This plasmid insertion was followed by an imprecise excision or recombination event, potentially mediated by the IS110 transposase or the pre/mob associated recombinase, whereby sites outside of the plasmid were recognised resulting in the excision of a region of the CTn containing tet $(\mathrm{L})$ and the pre/mob, rep and transposase genes but leaving orf6 and the full length orf 9 fusion behind, thus creating Tn916 (dotted box in Figure 4). Observations of imperfect excision events have been described previously. For example, Laverde Gomez et al. identified that fragments of the pathogenicity island of E. faecalis strain UW3114 remained in the chromosome following its excision. The authors concluded this imprecise excision occurred due to the erroneous recognition of sequences flanking the element that exhibited homology to internal sequences required for excision [38]. 


\subsection{Tn6079 Descends from the TT31 Tn916-Like Element}

Similarly, a relationship between Tn6079 and TT31 can be hypothesised where TT31 is an ancestral Tn916-like element to Tn6079 with the L. johnsonii Tn916-like element as an 'intermediate'. As previously described for the hypothetical evolution of Tn916, insertion of a plasmid containing pre /mob, rep, an IS110 transposase and orf6 genes and the $3^{\prime}-183 \mathrm{bp}$ of the full length orf9 inserts into orf9t of TT31, creating the 'intermediate' L. johnsonii Tn916-like element. Deletion of orf10, orf7, orf8, orf6 and orf9 then occurs following a recombination event between two regions of this CTn. This is similar to the event hypothesised by Croucher et al. to have resulted in the deletion of aphA-3 in a S. pneumoniae Tn916-like element [39]. Following this recombination event, an IS1216 element carrying erm(T) inserts upstream of orf5 giving rise to Tn6079 (Figure 5). It is also plausible that a lone IS1216 element inserted upstream of orf5 followed by the insertion of a second IS1216 type transposable unit containing erm(T). IS elements may flank accessory genes, including antibiotic resistance genes and can mediate their transposition; indeed IS1216 has been implicated in the dissemination of glycopeptide resistance genes and has been found flanking the $c f r$ resistance gene in the pEF-01 plasmids isolated from E. faecalis [40,41].

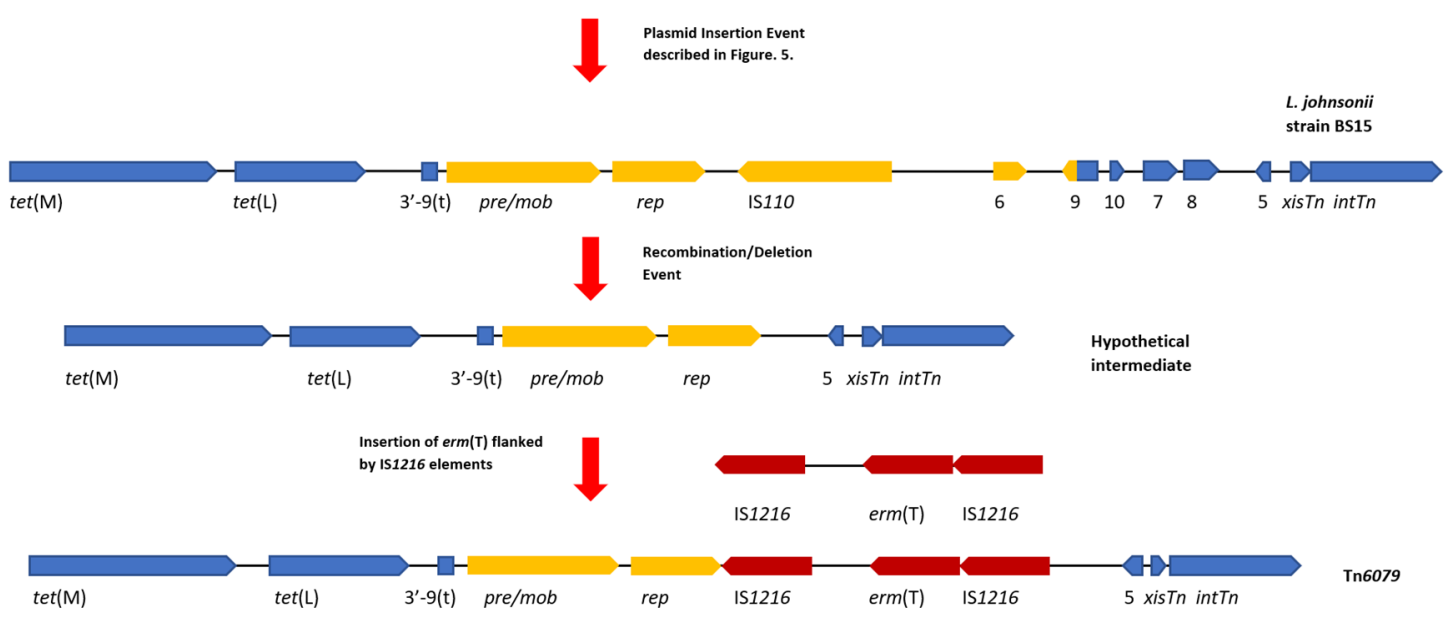

Figure 5. A diagram of the hypothetical events that created Tn6079. This hypothetical event assumes that the left $7226 \mathrm{bp}$ of TT31 are part of a larger Tn916-like element. Gene diagrams are not to scale. Following on from the insertion event that created the L. johnsonii strain BS15 'intermediate' Tn916-like element described in Figure 4, a deletion/recombination event results in the loss of orf6, orf9, orf10, orf7 and orf8, forming a hypothetical intermediate CTn. Insertion of erm(T) flanked by IS1216 elements (dark red) follows this deletion event creating Tn6079. For continuity with Figure 4, tet $(\mathrm{M}), \operatorname{tet}(\mathrm{L}), \operatorname{orf5}$, $x i s T n$ and intTn and the $3^{\prime}$ - end of orf9(t) are coloured blue and the plasmid associated genes are yellow.

\section{Conclusions}

In summary, we have identified a novel Tn916-like element from the human saliva metagenome using a functional metagenomic approach. As this Tn916-like element encoded an orf9 variant we have hypothesised that it is from an ancestral CTn to Tn916. These hypotheses point to a Tn916-like element with a gene organisation as described in TT31 as a predecessor of Tn916 CTns and that L. johnsonii strain BS15 encodes a representative of an intermediate Tn916-like element. The presence of the $3^{\prime}$-end remnant of orf9t in both L. johnsonii strain BS15 and Tn6079 supports the directionality of the events that we have described in this work. This directionality is further supported by the identification of homologs of orf6 and the $3^{\prime}$-end of the full length orf9 in a Staphylococcal pathogenicity island, which indicates that orf9 is a fusion gene. This work highlights the importance of orf9 as a point of evolutionary divergence in the Tn916/Tn1545 family of CTns.

Supplementary Materials: The following are available online at http://www.mdpi.com/2073-4425/11/5/548/s1, Table S1: Primers used in this study. Figure S1: TT31 Sequence. 
Author Contributions: L.J.R. and A.P.R. conceptualisation and sequence analyses. L.J.R. experimental work. L.J.R. curated the data and wrote the drafted manuscript. L.J.R., A.P.R. and M.F.A. contributed to the interpretation of data and reviewed and edited the manuscript. A.P.R. and M.F.A. acquired funding. All authors have read and agreed to publish this manuscript.

Funding: L.J.R. was jointly funded by a UCL IMPACT studentship at UCL (to A.P.R.) and the Seedcorn Programme at the Animal and Plant Health Agency (to M.F.A.).

Acknowledgments: We would like to thank Supathep Tansirichaiya (UCL) for providing us with the human saliva DNA that was used to construct the metagenomic library.

Conflicts of Interest: The authors declare no conflict of interest.

\section{References}

1. Ciric, L.; Mullany, P.; Roberts, A.P. Antibiotic and antiseptic resistance genes are linked on a novel mobile genetic element: Tn6087. J. Antimicrob. Chemother. 2011, 66, 2235-2239. [CrossRef] [PubMed]

2. Franke, A.; Clewell, D. Evidence for a chromosome-borne resistance transposon (Tn916) in Streptococcus faecalis that is capable of "conjugal" transfer in the absence of a conjugative plasmid. J. Bacteriol. 1981, 145, 494-502. [CrossRef] [PubMed]

3. Roberts, A.; Mullany, P. A modular master on the move: The Tn916 family of mobile genetic elements. Trends Microbiol. 2009, 17, 251-258. [CrossRef] [PubMed]

4. Wright, L.; Grossman, A. Autonomous Replication of the Conjugative Transposon Tn916. J. Bacteriol. 2016, 198, 3355-3366. [CrossRef]

5. Lunde, T.; Roberts, A.; Al-Haroni, M. Determination of copy number and circularization ratio of Tn916-Tn1545 family of conjugative transposons in oral streptococci by droplet digital PCR. J. Oral Microbiol. 2019, 11, 1552060. [CrossRef] [PubMed]

6. Cochetti, I.; Tili, E.; Mingoia, M.; Varaldo, P.; Montanari, M. erm(B)-carrying elements in tetracycline-resistant pneumococci and correspondence between Tn1545 and Tn6003. Antimicrob. Agents Chemother. 2008, 52, 1285-1290. [CrossRef]

7. Soge, O.; Beck, N.; White, T.; No, D.; Roberts, M.C. A novel transposon, Tn6009, composed of a Tn916 element linked with a Staphylococcus aureus mer operon. J. Antimicrob. Chemother. 2008, 62, 674-680. [CrossRef]

8. Flannagan, S.; Zitzow, L.; Su, Y.; Clewell, D. Nucleotide sequence of the 18-kb conjugative transposon Tn916 from Enterococcus faecalis. Plasmid 1994, 32, 350-354. [CrossRef]

9. Brenciani, A.; Bacciaglia, A.; Vecchi, M.; Vitali, L.; Varaldo, P.; Giovanetti, E. Genetic elements carrying $\operatorname{erm}(\mathrm{B})$ in Streptococcus pyogenes and association with tet(M) tetracycline resistance gene. Antimicrob. Agents Chemother. 2007, 51, 1209-1216. [CrossRef]

10. De Vries, L.; Vallès, Y.; Agersø, Y.; Vaishampayan, P.; García-Montaner, A.; Kuehl, J.; Christensen, H.; Barlow, M.; Francino, M. The gut as reservoir of antibiotic resistance: Microbial diversity of tetracycline resistance in mother and infant. PLoS ONE 2011, 6, e21644. [CrossRef]

11. Johnson, C.; Grossman, A. Integrative and Conjugative Elements (ICEs): What They Do and How They Work. Annu. Rev. Genet. 2015, 49, 577-601. [CrossRef] [PubMed]

12. McDougal, L.; Tenover, F.; Lee, L.; Rasheed, J.; Patterson, J.; Jorgensen, J.; LeBlanc, D. Detection of Tn917-like sequences within a Tn916-like conjugative transposon (Tn3872) in erythromycin-resistant isolates of Streptococcus pneumoniae. Antimicrob. Agents Chemother. 1998, 42, 2312-2318. [CrossRef] [PubMed]

13. Leon-Sampedro, R.; Fernandez-de-Bobadilla, M.; San Millan, A.; Baquero, F.; Coque, T. Transfer dynamics of Tn6648, a composite integrative conjugative element generated by tandem accretion of Tn5801 and Tn6647 in Enterococcus faecalis. J. Antimicrob. Chemother. 2019, 74, 2517-2523. [CrossRef] [PubMed]

14. Wang, H.; Mullany, P. The large resolvase TndX is required and sufficient for integration and excision of derivatives of the novel conjugative transposon Tn5397. J. Bacteriol. 2000, 182, 6577-6583. [CrossRef] [PubMed]

15. Brouwer, M.; Mullany, P.; Roberts, A.P. Characterization of the conjugative transposon Tn6000 from Enterococcus casseliflavus 664.1H1 (formerly Enterococcus faecium 664.1H1). FEMS Microbiol. Lett. 2010, 309, 71-76. [CrossRef] 
16. Sebaihia, M.; Wren, B.; Mullany, P.; Fairweather, N.; Minton, N.; Stabler, R.; Thomson, N.; Roberts, A.; Cerdeno-Tarraga, A.; Wang, H.; et al. The multidrug-resistant human pathogen Clostridium difficile has a highly mobile, mosaic genome. Nat. Genet. 2006, 38, 779-786. [CrossRef]

17. Rice, L.; Carias, L.; Marshall, S.; Rudin, S.; Hutton-Thomas, R. Tn5386, a novel Tn916-like mobile element in Enterococcus faecium D344R that interacts with Tn916 to yield a large genomic deletion. J. Bacteriol. 2005, 19, 6668-6677. [CrossRef]

18. Wozniak, R.; Fouts, D.; Spagnoletti, M.; Colombo, M.; Ceccarelli, D.; Garriss, G.; Dery, C.; Burrus, V.; Waldor, M. Comparative ICE genomics: Insights into the evolution of the SXT/R391 family of ICEs. PLoS Genet. 2009, 5, e1000786. [CrossRef]

19. Burrus, V.; Pavlovic, G.; Decaris, B.; Guédon, G. The ICESt1 element of Streptococcus thermophilus belongs to a large family of integrative and conjugative elements that exchange modules and change their specificity of integration. Plasmid 2002, 48, 77-97. [CrossRef]

20. Pavlovic, G.; Burrus, V.; Gintz, B.; Decaris, B.; Guedon, G. Evolution of genomic islands by deletion and tandem accretion by site-specific recombination: ICESt1-related elements from Streptococcus thermophilus. Microbiology 2004, 150, 759-774. [CrossRef]

21. Ciric, L.; Ellatif, M.; Sharma, P.; Patel, R.; Song, X.; Mullany, P.; Roberts, A. Tn916-like elements from human, oral, commensal streptococci possess a variety of antibiotic and antiseptic resistance genes. Int. J. Antimicrob. Agents 2012, 39, 360-361. [CrossRef] [PubMed]

22. Santoro, F.; Vianna, M.; Roberts, A. Variation on a theme; an overview of the Tn916/Tn1545 family of mobile genetic elements in the oral and nasopharyngeal streptococci. Front. Microbiol. 2014, 5, 535. [CrossRef] [PubMed]

23. Wade, W.; Thompson, H.; Rybalka, A.; Vartoukian, S. Uncultured Members of the Oral Microbiome. J. Calif. Dent. Assoc. 2016, 44, 447-456. [PubMed]

24. Reynolds, L.; Roberts, A.; Anjum, M. Efflux in the Oral Metagenome: The Discovery of a Novel Tetracycline and Tigecycline ABC Transporter. Front. Microbiol. 2017, 7, 1923. [CrossRef]

25. Tansirichaiya, S.; Reynolds, L.J.; Cristarella, G.; Wong, L.C.; Rosendahl, K.; Roberts, A.P. Reduced Susceptibility to Antiseptics Is Conferred by Heterologous Housekeeping Genes. Microb. Drug Resist. 2018, 24, 105-112. [CrossRef]

26. Roberts, A.; Mullany, P. Tn916-like genetic elements: A diverse group of modular mobile elements conferring antibiotic resistance. FEMS Microbiol. Rev. 2011, 35, 856-871. [CrossRef]

27. Zolezzi, P.; Cepero, P.; Ruiz, J.; Laplana, L.; Calvo, C.; Gómez-Lus, R. Molecular epidemiology of macrolide and tetracycline resistances in commensal Gemella sp. isolates. Antimicrob. Agents Chemother. 2007, 51, 1487-1490. [CrossRef]

28. Cerdá Zolezzi, P.; Laplana, L.; Calvo, C.; Cepero, P.; Erazo, M.; Gómez-Lus, R. Molecular basis of resistance to macrolides and other antibiotics in commensal viridans group streptococci and Gemella spp. and transfer of resistance genes to Streptococcus pneumoniae. Antimicrob. Agents Chemother. 2004, 48, 3462-3467. [CrossRef]

29. Olsvik, B.; Olsen, I.; Tenover, F. Detection of tet $(\mathrm{M})$ and tet $(\mathrm{O})$ using the polymerase chain reaction in bacteria isolated from patients with periodontal disease. Oral Microbiol. Immunol. 1995, 10, 87-92. [CrossRef]

30. Su, Y.; He, P.; Clewell, D. Characterization of the tet(M) determinant of Tn916: Evidence for regulation by transcription attenuation. Antimicrob. Agents Chemother. 1992, 36, 769-778. [CrossRef]

31. Celli, J.; Trieu-Cuot, P. Circularization of Tn916 is required for expression of the transposon-encoded transfer functions: Characterization of long tetracycline-inducible transcripts reading through the attachment site. Mol. Microbiol. 1998, 28, 103-117. [CrossRef] [PubMed]

32. Marra, D.; Scott, J. Regulation of excision of the conjugative transposon Tn916. Mol. Microbiol. 1999, 31, 609-621. [CrossRef]

33. Kumar, S.; Lekshmi, M.; Parvathi, A.; Ojha, M.; Wenzel, N.; Varela, M. Functional and Structural Roles of the Major Facilitator Superfamily Bacterial Multidrug Efflux Pumps. Microorganisms 2020, 8, 266. [CrossRef] [PubMed]

34. Roberts, M. Update on acquired tetracycline resistance genes. FEMS Microbiol. Lett. 2005, 245, $195-203$. [CrossRef] [PubMed]

35. Cotter, P.; Hill, C. Surviving the acid test: Responses of gram-positive bacteria to low pH. Microbiol. Mol. Biol. Rev. 2003, 67, 429-453. [CrossRef] [PubMed] 
36. Jin, J.; Guffanti, A.; Bechhofer, D.; Krulwich, T. Tet(L) and Tet(K) tetracycline-divalent metal/H+ antiporters: Characterization of multiple catalytic modes and a mutagenesis approach to differences in their efflux substrate and coupling ion preferences. J. Bacteriol. 2002, 184, 4722-4732. [CrossRef]

37. Wei, Y.; Deikus, G.; Powers, B.; Shelden, V.; Krulwich, T.; Bechhofer, D. Adaptive gene expression in Bacillus subtilis strains deleted for tetL. J. Bacteriol. 2006, 188, 7090-7100. [CrossRef]

38. Laverde Gomez, J.; Hendrickx, A.; Willems, R.; Top, J.; Sava, I.; Huebner, J.; Witte, W.; Werner, G. Intra- and interspecies genomic transfer of the Enterococcus faecalis pathogenicity island. PLoS ONE 2011, 29, e16720. [CrossRef]

39. Croucher, N.; Harris, S.; Fraser, C.; Quail, M.; Burton, J.; van der Linden, M.; McGee, L.; von Gottberg, A.; Song, J.; Ko, K.; et al. Rapid pneumococcal evolution in response to clinical interventions. Science 2011, 331, 430-434. [CrossRef]

40. Liu, Y.; Wang, Y.; Wu, C.; Shen, Z.; Schwarz, S.; Du, X.; Dai, L.; Zhang, W.; Zhang, Q.; Shen, J. First report of the multidrug resistance gene $c f r$ in Enterococcus faecalis of animal origin. Antimicrob. Agents Chemother. 2012, 56, 1650-1654. [CrossRef]

41. Xu, X.; Lin, D.; Yan, G.; Ye, X.; Wu, S.; Guo, Y.; Zhu, D.; Hu, F.; Zhang, Y.; Wang, F.; et al. vanM, a new glycopeptide resistance gene cluster found in Enterococcus faecium. Antimicrob. Agents Chemother. 2010, 54, 4643-4647. [CrossRef] [PubMed]

(C) 2020 by the authors. Licensee MDPI, Basel, Switzerland. This article is an open access article distributed under the terms and conditions of the Creative Commons Attribution (CC BY) license (http://creativecommons.org/licenses/by/4.0/). 